Rodrigo Freitas

BITTENCOURT

Antônio de Lisboa RIBEIRO

FILHO $^{2}$

Marcos Chalhoub Coelho

LIMA $^{2}$

Sidney Gonçalves Gonzalez

ALVES 2

Marta Freitas

VASCONCELOS ${ }^{3}$

Carmo Emanuel BISCARDE

Luciana da Silva LEAL $^{1}$

Eunice $\mathrm{OBA}^{1}$

Correspondências para:

Rodrigo F. Bittencourt - Departamento de Reprodução Animal e Radiologia, Faculdade de Medicina Veterinária e Zootecnia-UNESP, Botucatu-SP, 18618000, Brasil. Tel: (55) (14) 38116249 . Email:rfbvet@yahoo.com.br

Recebido para publicação: 20/06/2006 Aprovado para publicação: 24/04/2008

\title{
Efeito de um quelante de cálcio, um detergente e da lecitina de soja sobre a qualidade do sêmen caprino congelado-descongelado
}

\author{
1-Faculdade de Medicina Veterinária e Zootecnia da Universidade Estadual \\ Paulista, Botucatu-SP \\ 2-Escola de Medicina Veterinária da Universidade Federal da Bahia, \\ Salvador-BA \\ 3-Faculdade de Medicina Veterinária da Universidade de Brasília, Brasília-DF
}

\section{Resumo}

Nove amostras de sêmen de dois caprinos adultos, colhidas com a utilização de vagina artificial, foram submetidas a criopreservação, com o objetivo de verificar o efeito do Equex STM Paste e do EDTA, adicionados a um diluidor a base de Tris-gema de ovo, sobre a viabilidade espermática pós-descongelação. Também foi objetivo desse trabalho, avaliar a utilização de um diluidor comercial, a base de lecitina de soja (Bioexcell® - IMV, L’Aigle, França), para a congelação do sêmen caprino. Assim, foram formados cinco grupos experimentais: TRIS; TRIS+EDTA; TRIS+EQUEX; TRIS+EQUEX+EDTA e Bioexcell. Logo depois da avaliação, o sêmen foi diluído nos diferentes meios e envasado em palhetas de $0,25 \mathrm{~mL}$, com dose inseminante de $100 \times 10^{6}$ espermatozóides. As amostras foram submetidas ao resfriamento, com uma curva média de $0,46^{\circ} \mathrm{C} / \mathrm{min}$, até atingirem a temperatura de $5^{\circ} \mathrm{C}$, mantidas por mais $75 \mathrm{~min}$ em tempo de equilíbrio e, posteriormente, congeladas em nitrogênio líquido. A descongelação foi realizada em banho-maria a $37^{\circ} \mathrm{C}$ por 50 s. Não houve diferença ( $>0$,05) entre as médias de motilidade total e progressiva, em relação aos grupos TRIS, TRIS+EQUEX e TRIS+EQUEX+EDTA. O grupo Bioexcell obteve o menor $(\mathrm{P}<0,05)$ percentual de espermatozóides com motilidade total e progressiva após a descongelação. Após o teste de termoresistência, as melhores $(\mathrm{P}<0,05)$ taxas de motilidade total e progressiva foram observadas para os grupos com Equex STM (TRIS+EQUEX e TRIS+EQUEX+EDTA). Assim, pode-se afirmar que a adição do Equex promove uma maior manutenção das taxas de viabilidade espermática após a descongelação, quando comparado com os diluidores que não o continham.

\section{Introdução}

A criopreservação do sêmen promove uma elevação dos níveis de cálcio intracelular. ${ }^{1}$ Esse aumento resulta em disfunção e morte celular. ${ }^{1,2}$ Assim, visando minimizar o efeito deletério do cálcio durante o processo de congelação, a partir da década de 1970 alguns pesquisadores passaram a empregar o etileno-diamino-tetra-acetatodissódico (EDTA) em meios diluidores para a congelação de sêmen eqüino. ${ }^{3}$ Sua principal função seria quelar o cálcio no meio extracelular, diminuindo seu influxo para o meio intracelular ${ }^{4}$, inibindo, assim, o efeito deletério do cálcio sobre os espermatozóides ${ }^{2}$.

Em estudo com bovinos e bubalinos observou-se uma superioridade de $10-12 \%$ na taxa de motilidade espermática pósdescongelação com o diluidor que continha 0,1\% de EDTA em sua composição, em 
relação ao diluidor que não o continha. ${ }^{5}$ Resultados semelhantes foram verificados na espécie caprina, cuja motilidade espermática na descongelação foi $10 \%$ superior no diluidor com o EDTA $(0,1 \%)$ em sua composição. ${ }^{6}$

Alguns trabalhos têm demonstrado que a introdução de substâncias detergentes ao meio de congelação, como o Equex STM Paste (Nova Chemical Sales, Scituate Inc., MA, EUA), que tem como princípio ativo o dodecil-sulfato de sódio (DSS), tem melhorado os índices qualitativos, a sobrevivência, longevidade e fertilidade dos espermatozóides pós-descongelação. ${ }^{7,8,9} \mathrm{~A}$ função do DSS é aumentar a solubilidade dos fosfolipídios da gema de ovo, melhorando a disponibilidade e, assim, a sua capacidade de proteção à membrana plasmática dos espermatozóides contra o choque térmico e as alterações promovidas pelo processo de criopreservação. ${ }^{10}$

O Equex STM foi testado por Maia et al. ${ }^{7}$ no diluidor de congelação do sêmen ovino. Os autores compararam a congelabilidade do sêmen ovino em um diluidor a base de Tris-gema de ovo com $0 \%, 0,5 \%$ e com $1 \%$ de Equex STM. Esse estudo demonstrou que a adição do Equex aumentou $(\mathrm{P}<0,05)$ a motilidade total $\mathrm{e}$ progressiva dos espermatozóides em relação ao meio sem detergente. No diluidor Tris a motilidade total e progressiva foi de $34 \%$ e $24 \%$, respectivamente, enquanto que no meio Tris com $0,5 \%$ de Equex essas foram de $65 \%$ e $39 \%$ e no Tris com $1 \%$ foram $70 \%$ e $39 \%$, o que demonstra o efeito benéfico da utilização do detergente na congelação do sêmen ovino.

Os diluidores comumente utilizados para a congelação do sêmen caprino são a base dos aditivos de origem animal, como gema de ovo e/ou leite. Diversas formulações de meios contendo esses compostos têm sido estudadas para a congelação de sêmen dessa espécie como: tris-gema de ovo e gema de ovo-leite ${ }^{11} \mathrm{o}$ leite desnatado de vaca, em pó e reconstituído, citrato de sódio-glicose-gema de ovo, sacarose-EDTA, $\mathrm{CaNa}_{2}$-gema de ovo, rafinose-gema de ovo, Spermasol-gema de ovo, test-gema de ovo ${ }^{12}$, maltose-gema de ovo ${ }^{13}$, ácido cítrico-frutose-gema de ovo, com ou sem tris ${ }^{14}$, citrato-frutose-gema de ovo $^{15}$, leite de cabra ${ }^{16}$, água de coco-gema de ovo $^{17}$.

Apesar dos bons índices de fertilidade observados em estudos que utilizaram sêmen congelado em meio contendo gema de ovo e/ou leite ${ }^{11,18}$, estes componentes podem representar um risco potencial para a contaminação do diluidor, caso certos contaminantes (microbianos ou outros) estejam no produto in natura ${ }^{19}$. De fato, Bousseau et al. ${ }^{19}$ ao realizarem análises microbiológicas em dois diluidores de congelação de sêmen comerciais, adicionados de gema de ovo ou esta associada ao leite, demonstraram que todas as amostras estudadas $(n=17)$, mesmo na presença de associações de antibióticos de larga ação (penicilina, estreptomicina, lincomicina, espectinomicina ou gentamicina, tilosina, lincomicina e espectinomicina) encontravamse com contaminação moderada (10-60 $\mathrm{CFU} / \mathrm{mL}$ ) de agentes bacterianos ou destes associados à mycoplasma. Este fator associado a falta de padronização desses aditivos, que podem apresentar grande variação nas suas composições ${ }^{20}$ estimularam o desenvolvimento de diluidores comerciais formulados a base de lecitina de soja, como uma alternativa à utilização da gema de ovo e do leite $\left(\right.$ Biociphos $^{\circledR}$ and Bioexcell ${ }^{\circledR}$, IMV, L'Aigle, France) e com índices de fertilidade semelhantes àqueles do sêmen de bovinos $\mathrm{e}$ ovinos criopreservados com os meios convencionais ${ }^{21,22}$. Dessa forma, os diluidores sem os componentes de origem animal podem ser uma alternativa segura, quando o sêmen congelado for utilizado para a introdução de um novo material genético em um rebanho ou em um país. ${ }^{20}$

Mesmo com bons resultados obtidos com a utilização do Equex STM Paste e do diluidor comercial Bioexcell ${ }^{\circledR}$, para a criopreservação do sêmen de outras espécies, nenhuma citação foi encontrada para a espécie caprina. Assim, este estudo teve como objetivos avaliar o efeito da associação do 
Equex STM e do EDTA à um diluidor a base de Tris-gema de ovo e compará-lo à eficácia de um diluidor comercial, a base de lecitina de soja $\left(\right.$ Bioexcell $^{\circledR}$ ) e sem aditivos de origem animal, na congelação do sêmen caprino.

\section{Material e Método}

\section{Preparo dos diluidores}

A solução de Tris-gema de ovoglicerol $^{23}$ foi o diluidor base utilizado neste trabalho. Todos os reagentes químicos que o compuseram $(2,42 \mathrm{~g}$ de Tris, $1,5 \mathrm{~g}$ de ácido cítrico, $1,25 \mathrm{~g}$ de glicose e $6 \%$ de glicerol) exceto o sulfato de gentamicina $(13,4 \mathrm{mg})$ (Schering-Plough S/A, São Paulo-SP, Brasil), foram fabricadas pelo laboratório VETEC Ltda (Duque de Caxias-RJ, Brasil), bem como o EDTA. Para o desenvolvimento do experimento foram formados cinco grupos experimentais: Grupo 1- TRIS; Grupo 2- TRIS+EDTA; Grupo 3TRIS+EQUEX; Grupo 4- TRIS + EQUEX+ EDTA e Grupo 5- Bioexcell.

Aos diluidores TRIS+EDTA e TRIS+EQUEX+EDTA foi adicionado $0,1 \%$ de EDTA e aos grupos TRIS+EQUEX e TRIS+EQUEX+EDTA foi acrescentado 0,5\% de Equex STM (Nova Chemical Sales, Inc., MA, EUA). O diluidor comercial Bioexcell ${ }^{(I M V, ~ L ' A i g l e, ~ F r a n c ̧ a), ~}$ a base de lecitina de soja, compôs o último protocolo experimental.

\section{Processamento do sêmen}

Nove amostras de sêmen de dois caprinos adultos, selecionados após a avaliação clínica e exame andrológico, foram utilizadas nesse experimento. Logo após as colheitas por vagina artificial, realizadas em dias alternados, o sêmen de cada reprodutor era encaminhado ao laboratório de processamento, mantido em banho-maria à temperatura de $35^{\circ} \mathrm{C}$ e avaliado quanto ao volume, cor, aspecto, motilidade total e progressiva, vigor, turbilhonamento e concentração. Também foram retiradas alíquotas do sêmen para avaliação dos defeitos espermáticos através de microscopia de contraste de fase com aumento de 1000x. Após as avaliações iniciais e cálculo da concentração espermática, um total de $400 \times 10^{6}$ de espermatozóides com motilidade progressiva eram adicionados a tubos pré-aquecidos contendo $1 \mathrm{~mL}$ de cada um dos cinco diluidores, já com o percentual final de glicerol (diluição em uma etapa), compondo quatro doses inseminantes de $100 \times 10^{6} \mathrm{de}$ espermatozóides com motilidade progressiva/0,25mL, para cada grupo experimental. Após a diluição nos diferentes meios, o sêmen foi novamente avaliado, envasado em palhetas de $0,25 \mathrm{~mL}$ e resfriado até $5^{\circ} \mathrm{C}$ em câmara refrigerada com temperatura estabilizada, a uma taxa de resfriamento médio de $0,46^{\circ} \mathrm{C} / \mathrm{min}$, previamente controlada através de um termômetro digital de haste fina. Depois de $75 \mathrm{~min}$ de equilíbrio à temperatura de $5^{\circ} \mathrm{C}$, a congelação foi efetuada mantendo-se as palhetas por $20 \mathrm{~min}$ em vapor de nitrogênio líquido e posteriormente mergulhadas no nitrogênio líquido. As amostras foram descongeladas em banho-maria à $37^{\circ} \mathrm{C}$ por 50 segundos, cinco dias depois da congelação.

Avaliação do sêmen após a descongelação e teste de termoresistência Logo após a descongelação as amostras foram avaliadas quanto a motilidade total, progressiva, vigor espermático e submetidas ao teste de termoresistência (TTR), conforme descrito pelo Colégio Brasileiro de Reprodução Animal (CBRA). ${ }^{24} \mathrm{O}$ sêmen descongelado também foi avaliado quanto ao percentual de defeitos espermáticos presentes em 200 células, utilizando para tanto a microscopia de contraste de fase com aumento de 1000x e a classificação espermática preconizada pelo CBRA. ${ }^{24}$

Delineamento e análise estatística Cada reprodutor foi considerado um bloco e o delineamento estatístico foi inteiramente casualizado, no qual os ejaculados foram considerados repetições e 
os protocolos de processamento do sêmen foram considerados tratamentos. Os cálculos de média, desvio-padrão e análise de variância foram realizados conforme Sampaio ${ }^{25}$. Para análise estatística das características avaliadas foi empregado o pacote estatístico Statistical Analysis System (SAS) - versão $5.0^{26}$, com a seguinte sequência de análises:

1- A consistência dos dados e a análise descritiva (médias e desvio-padrão) das características de interesse ao estudo foram realizadas mediante o emprego do Procedimento MEANS.

2- As variáveis (defeitos maiores, defeitos menores, defeitos de acrossoma e defeitos totais; motilidade espermática total e progressiva e vigor espermático) foram comparadas entre os tratamentos por meio do Procedimento GLM, utilizando-se o teste de Student Newman Keuls (SNK) com o nível de significância de 5\%.

\section{Resultados}

Os ejaculados utilizados neste estudo tiveram como características um volume total de $0,57 \pm 0,20$ e concentração espermática de $5,25 \pm 1,76$ × $10^{9}$ espermatozóides/mL. Como se pode observar na tabela 1 , o processo de criopreservação afetou significativamente $(\mathrm{P}<0,05)$ as taxas de motilidade espermática total e progressiva em todos os diluidores testados, em relação ao sêmen fresco. Contudo, não foi verificada diferença significativa entre o vigor do sêmen fresco e nos diluidores TRIS, TRIS+EQUEX e TRIS+EQUEX+EDTA, logo após a descongelação.

Em geral, todos os diluidores, exceto o Bioexcell, apresentaram valores médios de motilidade progressiva e vigor, acima dos valores mínimos recomendados para o sêmen caprino descongelado, de 30\% para motilidade progressiva e 2 para vigor, segundo normas do $\mathrm{CBRA}^{24}$, resultados esses que se repetiram após o teste de termoresistência (TTR) (Tabela 2).

Pode-se verificar também que após o TTR os diluidores que continham o Equex STM (TRIS+EQUEX e TRIS+EQUEX+EDTA) obtiveram valores médios para a motilidade total e progressiva, semelhantes entre si $(\mathrm{P}>0,05)$ e superiores $(\mathrm{P}<0,05)$ em relação a todos os demais grupos experimentais, e o Bioexcell proporcionou os resultados mais inferiores $(\mathrm{P}<0,05)$ tanto na descongelação, como após o TTR, para os parâmetros MT, MP e VIGOR (Tabelas 1 e 2).

As médias de defeitos menores, defeitos maiores e defeitos totais, observados para a maioria dos diluidores estudados (Tabela 3), estiveram abaixo dos valores máximos recomendados pelo $\mathrm{CBRA}^{24}$ para o sêmen caprino descongelado, de $20 \%$ para o número de defeitos totais e $10 \%$ de defeitos maiores. Apenas o grupo TRIS superou a média de defeitos totais $(20,55 \%)$.

Apesar das diferenças nos percentuais médios de defeitos maiores encontrados para os espermatozóides congelados nos diversos diluidores, as mesmas não foram significativas. Entretanto, levando-se em consideração apenas os valores numéricos, pode-se verificar que os diluidores TRIS+EQUEX+EDTA e TRIS+EQUEX obtiveram os menores percentuais de defeitos maiores e defeitos de acrossoma e, assim como o TRIS+EDTA, promoveram índices semelhantes $(\mathrm{P}>0,05)$ de lesões acrossomais em relação ao sêmen fresco, o que pode sugerir a eficácia desses diluidores para a preservação da morfologia espermática durante o processo de congelação-descongelação. Já para os diluidores TRIS e Bioexcell ${ }^{\circledR}$ verificaram-se maiores $(\mathrm{P}<0,05)$ índices de defeitos acrossomais quando comparados ao ejaculado fresco.

\section{Discussão}

A adição de EDTA ao diluidor não melhorou as taxas de motilidade espermática total, progressiva e vigor espermático pósdescongelação e após o TTTR. Achados semelhantes ao deste estudo foram descritos por Aisen et al. ${ }^{27}$ em ovinos, que não 
Tabela 1 - Médias e desvios-padrão de motilidade total (MT), motilidade progressiva (MP) e vigor espermático no sêmen fresco, comparativamente aos cinco diluidores (TRIS, TRIS + EDTA, TRIS + EQUEX, TRIS + EDTA + EQUEX, Bioexcell), logo após a descongelação

\begin{tabular}{cccc}
\hline TRATAMENTO & $\begin{array}{c}\text { MT } \\
(\text { Média } \pm \text { S) }\end{array}$ & $\begin{array}{c}\text { MP } \\
(\text { Média } \pm \text { S) }\end{array}$ & $\begin{array}{c}\text { VIGOR } \\
(\text { Média } \pm \text { S) }\end{array}$ \\
\hline Sêmen Fresco & $61,00 \pm 7,40^{\mathrm{a}}$ & $55,55 \pm 7,26^{\mathrm{a}}$ & $3,94 \pm 0,16^{\mathrm{a}}$ \\
TRIS & $38,75 \pm 6,94^{\mathrm{bc}}$ & $34,37 \pm 6,23^{\mathrm{bc}}$ & $3,31 \pm 0,45^{\mathrm{ab}}$ \\
TRIS+EDTA & $35,71 \pm 6,07^{\mathrm{c}}$ & $30,71 \pm 6,07^{\mathrm{c}}$ & $2,92 \pm 0,53^{\mathrm{b}}$ \\
TRIS+EQUEX & $44,37 \pm 4,95^{\mathrm{b}}$ & $39,37 \pm 4,95^{\mathrm{b}}$ & $3,64 \pm 0,37^{\mathrm{a}}$ \\
TRIS+EQUEX +EDTA & $43,75 \pm 6,40^{\mathrm{b}}$ & $39,37 \pm 6,23^{\mathrm{b}}$ & $3,31 \pm 0,53^{\mathrm{ab}}$ \\
Bioexcell & $27,14 \pm 4,87^{\mathrm{d}}$ & $21,42 \pm 6,23^{\mathrm{d}}$ & $2,14 \pm 0,62^{\mathrm{c}}$ \\
\hline
\end{tabular}

Valores seguidos de letras minúsculas distintas na mesma coluna diferem entre si $(\mathrm{P}<0,05)$

Tabela 2 - Médias e desvios-padrão de motilidade total (MT), motilidade progressiva (MP) e vigor espermático nos cinco diluidores (TRIS, TRIS + EDTA, TRIS + EQUEX, TRIS + EDTA + EQUEX e Bioexcell), logo após o teste de termoresistência (TTR)

\begin{tabular}{|c|c|c|c|}
\hline TRATAMENTO & $\begin{array}{c}\text { MT } \\
(\text { Média } \pm \text { S) }\end{array}$ & $\begin{array}{c}\text { MP } \\
(\text { Média } \pm \mathrm{S})\end{array}$ & $\begin{array}{c}\text { VIGOR } \\
(\text { Média } \pm \text { S) }\end{array}$ \\
\hline TRIS & $35,62 \pm 5,62^{b}$ & $31,87 \pm 5,93^{b}$ & $3,08 \pm 0,49^{\mathrm{bc}}$ \\
\hline TRIS+EDTA & $33,57 \pm 4,75^{b}$ & $30,00 \pm 5,00^{b}$ & $2,71 \pm 0,48^{\mathrm{c}}$ \\
\hline TRIS+EQUEX & $43,75 \pm 3,53^{\mathrm{a}}$ & $39,37 \pm 4,95^{\mathrm{a}}$ & $3,81 \pm 0,25^{\mathrm{a}}$ \\
\hline TRIS+EQUEX +EDTA & $43,12 \pm 7,03^{\mathrm{a}}$ & $38,12 \pm 7,03^{\mathrm{a}}$ & $3,50 \pm 0,46^{\mathrm{ab}}$ \\
\hline Bioexcell & $25,71 \pm 4,49^{\mathbf{c}}$ & $20,71 \pm 4,49^{c}$ & $1,92 \pm 0,67^{\mathrm{d}}$ \\
\hline
\end{tabular}

Valores seguidos de letras minúsculas distintas na mesma coluna diferem entre si $(\mathrm{P}<0,05)$.

Tabela 3 - Médias de defeitos menores (DME), defeitos maiores (DM), defeitos de acrossoma (AC) e defeitos espermáticos totais (DT) do sêmen fresco, e após a descongelação nos cinco diluidores (TRIS, TRIS + EDTA, TRIS + EQUEX, TRIS + EQUEX+EDTA e Bioexcell)

\begin{tabular}{|c|c|c|c|c|}
\hline TRATAMENTO & $\begin{array}{c}\text { DME } \\
(\text { Média } \pm \text { S) }\end{array}$ & $\begin{array}{c}\text { DM } \\
(\text { Média } \pm \text { S) }\end{array}$ & $\begin{array}{c}\mathbf{A C} \\
(\text { Média } \pm \mathrm{S})\end{array}$ & $\begin{array}{c}\text { DT } \\
(\text { Média } \pm \text { S) }\end{array}$ \\
\hline Sêmen Fresco & $07,77 \pm 3,53^{\mathrm{ab}}$ & $2,11 \pm 1,38$ & $0,05 \pm 0,16^{\mathrm{a}}$ & $09,94 \pm 4,33^{\mathrm{a}}$ \\
\hline TRIS & $14,77 \pm 8,99^{b}$ & $5,77 \pm 4,60$ & $2,66 \pm 2,82^{b}$ & $20,55 \pm 11,40^{b}$ \\
\hline TRIS+EDTA & $07,12 \pm 5,02^{\mathrm{ab}}$ & $4,50 \pm 2,67$ & $1,25 \pm 1,48^{\mathbf{a b}}$ & $10,37 \pm 5,65^{\mathrm{a}}$ \\
\hline TRIS+EQUEX & $08,44 \pm 7,43^{\mathrm{ab}}$ & $2,44 \pm 1,87$ & $1,11 \pm 1,36^{\mathbf{a b}}$ & $10,88 \pm 8,35^{\mathrm{a}}$ \\
\hline TRIS+EQUEX+EDTA & $05,50 \pm 4,24^{\mathrm{a}}$ & $2,87 \pm 2,53$ & $0,37 \pm 0,51^{\mathbf{a b}}$ & $08,37 \pm 5,04^{\mathrm{a}}$ \\
\hline Bioexcell & $10,37 \pm 5,31^{\mathrm{ab}}$ & $4,25 \pm 2,37$ & $2,50 \pm 2,50^{\mathrm{b}}$ & $14,12 \pm 6,77^{\mathrm{ab}}$ \\
\hline
\end{tabular}

Valores seguidos de letras minúsculas distintas na mesma coluna diferem entre si $(\mathrm{P}<0,05)$.

encontraram diferença significativa para a motilidade espermática pós-descongelação entre o sêmen diluído com Tris-gema de ovo $(58,5 \%)$, e o que continha $0,1 \%$ de EDTA associado ao diluidor $(52,1 \%)$.

Apesar da semelhança $(P>0,05)$ observada entre os índices de motilidade espermática total, motilidade progressiva e vigor espermático, foi verificado um melhor desempenho do diluidor TRIS+EDTA em relação ao TRIS $(\mathrm{P}<0,05)$, para o número de defeitos espermáticos totais (Tabela 3). O melhor índice de integridade da morfologia espermática no diluidor com EDTA é justificado já que este, ao quelar o cálcio, minimiza a sua biodisponibilidade no meio extracelular, reduzindo o seu influxo e inibindo, assim, o desenvolvimento das lesões espermáticas provocadas por esse processo $\mathrm{S}^{4,5}$, altamente relacionado à diminuição da viabilidade e morte celular, durante a congelação ${ }^{2}$. De forma contrária, mas utilizando o mesmo percentual de EDTA em diuidor Tris-gema de ovo, Bittencourt et al. ${ }^{6}$ observaram uma maior $(\mathrm{P}<0,05)$ taxa de caudas fortemente dobradas 
(49,5\% versus $26 \%$ ) e de caudas com dobramento simples (64,5\% versus 38,2\%.)

Essas divergências observadas na literatura científica, em relação a eficácia da utilização do EDTA para a criopreservação espermática, está relacionada a ocorrência de alguns fenômenos, e entre estes o principal seria o fator de interação entre os componentes do meio diluidor, uma vez que nem sempre a remoção do cálcio do meio extracelular é desejável. Este fato foi observado em estudo que, ao avaliar o efeito da associação EDTA e trealose ${ }^{28}$, demonstrou que o diluidor com EDTA promoveu maiores índices de alterações da membrana espermática, bem como maiores níveis de peroxidação lipídica. Os autores concluíram que a ação antioxidante da trealose foi inibida pela retirada do cálcio do meio extracelular pelo EDTA, sugerindo a existência de um efeito positivo da interação entre a trealose e o cálcio, sobre a região polar dos fosfolipídios da membrana espermática. No entanto, no presente estudo, a remoção do cálcio do meio extracelular promoveu efeito desejável sobre os espermatozóides, comprovado pelo menor índice $(\mathrm{P}<0,05)$ de defeitos espermáticos totais no sêmen congelado com o diluidor TRIS+EDTA (Tabela 3).

Apesar da inexistência de relatos da utilização do Equex STM em diluidores para a criopreservação do sêmen caprino, os resultados obtidos neste experimento com os grupos que continham esse detergente, confirmam estudos anteriores realizados com outras espécies, que demonstraram o seu efeito positivo para a manutenção dos bons parâmetros espermáticos pósdescongelação. ${ }^{7,8,9,10}$ Os melhores índices de motilidade total e progressiva, verificados para os diluidores contendo Equex STM após o TTR, corroboram com os resultados citados por Peña e Linde-Forsberg ${ }^{8}$ e Rota et al. ${ }^{10}$, que comparando o efeito da adição do Equex ao diluidor de sêmen canino, obtiveram melhores índices de sobrevivência após a descongelação e o TTR. Maia et al. ${ }^{7}$ confirmaram estas observações, relatando taxas superiores $(\mathrm{P}<0,05)$ de motilidade progressiva no diluidor de sêmen ovino com o Equex STM (39\% versus 24\%). Esses achados podem ser explicados pois, o detergente dodecil-sulfato de sódio (DDS) que é a substância ativa do Equex STM Paste tem, como função primordial, aumentar a disponibilidade dos fosfolipídios da gema de ovo, melhorando a sua capacidade de proteção à membrana plasmática dos espermatozóides contra o choque térmico, minimizando o surgimento das alterações promovidas pelo processo de criopreservação. ${ }^{10}$ Este efeito é confirmado pelo fato que os diluidores com Equex em sua composição, além das melhores taxas de motilidade espermática, apresentaram os menores percentuais numéricos de defeitos maiores, menores, de acrossoma e defeitos totais, em relação a todos os outros diluidores estudados (Tabela 3). Os baixos índices de viabilidade espermática obtidos neste trabalho com o diluidor Bioexcell discordam dos resultados satisfatórios relatados em trabalhos que utilizaram esse diluidor, ou outro a base de leticina de soja, para criopreservação do sêmen de ovinos ${ }^{20,22}$ e bovinos ${ }^{21,29}$. Gil et al. ${ }^{22}$, utilizando o Bioexcell ${ }^{\circledR}$ em ovinos, encontraram médias de motilidade espermática pósdescongelação de $56 \%$, valor semelhante $(\mathrm{P}>0,05)$ aos $58 \%$ verificados para o diluidor a base de leite-gema de ovo testado pelos autores e superior aos $27,14 \%$ encontrados neste experimento com Bioexcell. Resultados semelhantes foram relatados pela mesma equipe de pesquisa em outro estudo. ${ }^{20}$ Contudo, em ambos os trabalhos foi utilizada a metodologia rotineiramente empregada na Suécia com a diluição do sêmen em duas etapas, adicionando a fração do diluidor com glicerol somente após o resfriamento do sêmen a temperatura de $5^{\circ} \mathrm{C}$, tanto para o diluidor Bioexcell quanto para o leite-gema de ovo. No presente estudo, para todos os grupos experimentais foi utilizado o método de diluição em uma etapa, já com a concentração final de glicerol, inclusive para o Bioexcell, já que esta é a forma na qual é feita a sua comercialização no Brasil. Esse fato sugere que melhores 
resultados com a utilização do Bioexcell para a congelação do sêmen caprino poderiam ser obtidos empregando-se a diluição em duas etapas.

A partir das avaliações in vitro, podese concluir que a adição do Equex STM Paste ao diluidor Tris-gema de ovo promove uma maior manutenção da viabilidade dos espermatozóides de caprinos pós-descongelação, quando comparado com os diluidores que não o contem. Da mesma forma, o EDTA mostra-se eficaz na preservação da morfologia espermática pós-descongelação. No entanto, estudos de fertilidade in vivo, com a incorporação desses componentes ao diluidor, devem ser realizados para efetiva comprovação de sua eficácia na congelação do sêmen caprino. $\mathrm{O}$ diluidor comercial Bioexcell foi ineficaz, sugerindose a realização de novos estudos para avaliar o efeito do método de diluição (uma ou duas etapas) sobre a congelabilidade dos espermatozóides caprinos.

\section{Agradecimentos}

Esse trabalho foi desenvolvido com o apóio financeiro da CAPES e da FAPESB e patrocínio do laboratório Tecnopec.

\title{
Effects of a calcium chelator, a detergent and the soybean lecithin on the quality of the frozen-thawed goat semen
}

\begin{abstract}
The aim of this paper was verifying the effect of the Equex STM Paste and EDTA addition to a Tris-egg yolk extender, on the postthaw goat sperm viability. Nine semen samples of two adult goats were collected by artificial vagina and cryopreserved. It was also objective of this study, to evaluate the utilization of a soybean lecithin based commercial extender (Bioexcell ${ }^{\circledR}$ - IMV, L'Aigle, French) for the goat semen freezing. They were formed five experimental groups: TRIS; TRIS+EDTA; TRIS+EQUEX; TRIS+EDTA+EQUEX e Bioexcell. After evaluation, the semen was diluted in the five extenders and packed in $0.25 \mathrm{~mL}$ straws with 100 million of motile spermatozoa. The samples were cooled at $0,46^{\circ} \mathrm{C} / \mathrm{min}$ to $5^{\circ} \mathrm{C}$, submitted at $75 \mathrm{~min}$ of equilibration time and frozen in liquid nitrogen vapour. The thawing was accomplished in $37^{\circ} \mathrm{C}$ water bath for 50 s. There were no differences $(\mathrm{P}>0,05)$ on the means of post-thaw total and progressive sperm motility among the groups TRIS, TRIS+EQUEX and TRIS+EQUEX+EDTA. The Bioexcell group obtained the least $(\mathrm{P}<0,05)$ percentage of post-thawing total and progressive sperm motility. After the thermotolerance test, it was observed the greatest $(\mathrm{P}<0,05)$ rates of total and progressive sperm motility in the Equex STM groups (TRIS+EQUEX and TRIS+EQUEX+EDTA). Thus, it can be affirmed that the Equex addition promotes better maintenance rates in the pos-thaw sperm viability, when compared with the extenders that did not contain it.
\end{abstract}

Key word:

Goat.

Semen. Cryopreservation.

\section{Referências}

1 WATSON, P. F. The causes of reduced fertility with cryopreserved semen. Animal Reproduction Science, v. 60-61, p. 482-492, 2000.

2 AMANN, R. P.; PICKETT, B. W. Principle of cryopreservation and a review of stallion spermatozoa. Equine Veterinary Science, v. 7, n. 3, p. 145-174, 1987.

3 MARTIN, J. C.; KLUG, E.; GÜNZEL, A. R. Centrifugation of stallion semen and storage in large volumes straws. Journal of Reproduction and Fertility Supplement, v. 27, p. 47-51, 1979.

4 WATSON, P. F. The effects of cold shock on sperm 
cell membranes. In: MORRIS, G. J.; CLARKE, A. (Ed.) Effects of low temperatures on biological membranes. London: Academic Press, 1981. p. 189.

5 DHAMI, A. J.; SAHNI, K. L. S. O. Effect of extenders, additives and deep freezing on the leakage of lactic dehydrogenase from cattle and buffalo spermatozoa. Indian Journal of Animal Sciences, v. 63, n. 3, p. 251 . 256, 1993.

6 BITTENCOURT, R. F. et al. Utilização de glicerol ou etilenoglicol como crioprotetores na congelação de sêmen caprino. Ciência Animal Brasileira, v. 5, n. 1, p. 27-32, 2004.

7 MAIA, M. S. et al. Efeito da adição do Equex-STM ao diluente Tris-gema na motilidade do espermatozóide criopreservado de carneiro. Acta Scientiae Veterinariae, v. 33, p. 311, 2005. Suplemento 1.

8 PEÑA, A.; LINDE FORSBERG, C. Effects of equex, one or two step diluition, and two freesing and thawing rates on post-thaw survival of dog spermatozoa. Theriogenology, v. 54, n. 6, p. 859-875, 2000.

9 PEÑA, A. et al. Effects of Equex from different sources on post-thaw survival, longevity and intracellular $\mathrm{Ca}^{2+}$ concentration of dog spermatozoa. Theriogenology, $\mathrm{v}$. 59, n. 8, p. 1725-1739, 2003.

10 ROTA, A. et al. Effects of equex STM paste on viability of frozen-thawed dog spermatozoa during in vitro incubation at $38{ }^{\circ} \mathrm{C}$. Theriogenology, v. 47, p. 1093$1101,1997$.

11 DORADO, J.; RODRÍGUEZ, I.; HIDALGO, M. Cryopreservation of goat spermatozoa: Comparison of two freezing extenders based on post-thaw sperm quality and fertility rates after artificial insemination. Theriogenology, v. 68, n. 2, p. 168-177J, 2007.

12 LEBOEUF, B.; RESTALL, B.; SALAMON, S Production and storage of goat semen for artificial insemination. Animal Reproduction Science, v. 62, p. 113-141, 2000

13 DAS, K. K.; RAJKONWAR, C. K. Acrosomal changes of buck spermatozoa after equilibration and freezing in egg yolk citrate glycerol extender. Indian Veterinary Journal, v. 73, n. 1, p. 35-40, 1996.

14 SINGH, L. P.; PURBEY, L. N. Effect of ultra-low temperature on acrosomal integrity of buck spermatozoa in Tris and citrate dilutors. Indian Journal of Animal Reproduction, v. 17, n. 1, p. 45-49, 1996.

15 SINGH, L. P.; PURBEY, L. N. Preservability of goat spermatozoa in Tris an citrate extenders at $-196^{\circ} \mathrm{C}$ and $5^{\circ} \mathrm{C}$. Indian Journal of Animal Sciences, v. 66, n. 11, p. 1139-1141, 1996.

16 DESHPANDE, S. B.; MEHTA, V. M. Effect of dilutors and different glycerol levels on pre-freeze and postfreeze sperm motility and live sperm count in Surti buck semen. Indian Journal of Animal Sciences, v. 61, n. 10 , p. 1093-1095, 1991

17 ARAÚJO, A. A. de; NUNES, J. F. Utilização da água de côco in natura com adição de ovo como diluidor de congelação de sêmen caprino. In: CONGRESSO BRASILEIRO DE REPRODUÇÃO ANIMAL, 9, 1991, Belo Horizonte. Anais... Belo Horizonte: Colégio Brasileiro de Reprodução Animal. 1991, v. 2, p. 435.

18 PETERSON, K. et al. Microscopic and flow cytometric semen assessment of Dutch Al-bucks: Effect of semen processing procedures and their correlation to fertility. Theriogenology, v. 67, n. 4, p. 863-887, 2007.

19 BOUSSEAU, S. et al. Comparison of bacteriological qualities of various egg yolk sources and the in vitro and in vivo fertilizing potential of bovine semen frozen in egg yolk or lecithin-based diluents. Theriogenology, v. 50, p. 699-706, 1998.

$20 \mathrm{GIL}$, J. et al. Fertility of ram semen frozen in Bioexcell and used for cervical artificial insemination. Theriogenology, v. 59, n. 5-6, p. 1157-1170, 2003.

$21 \mathrm{GIL}$, J. et al. Functional sperm parameters and fertility of bull semen extended in Biociphos Plus ${ }^{\circ}$ and Trilady ${ }^{\circ}$. Reproduction in Domestic Animals, v. 35, p. 69-77, 2000.

$22 \mathrm{GIL}$, J et al. Influence of extender, temperature, and addition of glycerol on post-thaw sperm parameters in ram semen. Theriogenology, v. 59, n. 5-6, p. 12411255,2003

23 ROBERTS, S. J. Veterinary obstetrics and genital diseases (Theriogenology). 3. ed. Michigan: Edwards Brothers, 1986. 981 p.

24 HENRY, M.; NEVES, J. P. Manual para exame andrológico e avaliação de sêmen animal. 2. ed. Belo Horizonte: Colégio Brasileiro de Reprodução Animal, 1998. 49 p.

25 SAMPAIO, I. B. M. Estatística aplicada à experimentação animal. Belo Horizonte: FEPMVZ, 1998. 221 p.

26 SAS. User's guide: statistcs, version 5. Cary: SAS Institute, 1996. 956 p.

27 AISEN E. G. et al. Effect of trehalose and edta on cryoprotective action of ram semen diluents. Theriogenology, v. 53, p. 1053-1061, 2000.

28 AISEN, E. G. et al. Ultramicroscopic and biochemical changes in ram spermatozoa cryopreserved with trehalose-based hypertonic extenders. Cryobiology, v. 50, n. 3 , p. 239-249, 2005.

29 VAN WAGTENDONK DE LEEW, A. M. et al. Fertility results using bovine semen cryopreserved with extenders based on egg yolk and soy-bean extract. Theriogenology, v. 54, p. $57-67,2000$. 